\title{
Bioinspired Strategy for Controlled Polymerization and Photopatterning of Plant Polyphenols
}

\author{
Farid Behboodi-Sadabad, ${ }^{\dagger,}$ \\ and Pavel A. Levkin*,t, \\ Huijie Zhang, ${ }^{\S}$ Vanessa Trouillet, ${ }^{\prime, \perp}$ Alexander Welle, ${ }^{\perp, \#}$ Nicolas Plumeré, ${ }^{\S}$ \\ ${ }^{\dagger}$ Institute of Organic Chemistry, Karlsruhe Institute of Technology, 76021 Karlsruhe, Germany \\ ${ }^{\ddagger}$ Institute of Toxicology and Genetics, Karlsruhe Institute of Technology, 76344 Eggenstein Leopoldshafen, Germany \\ ${ }^{\S}$ Center for Electrochemical Sciences, Ruhr Universität Bochum, D 44780 Bochum, Germany \\ "Institute for Applied Materials, Karlsruhe Institute of Technology, 76344 Eggenstein Leopoldshafen, Germany \\ ${ }^{\perp}$ Karlsruhe Nano Micro Facility, Karlsruhe Institute of Technology, 76344 Eggenstein Leopoldshafen, Germany \\ \#Institute of Functional Interfaces, Karlsruhe Institute of Technology, 76344 Eggenstein Leopoldshafen, Germany
}

\begin{abstract}
Plant derived polyphenols have been widely used to design new multifunctional materials for particle and surface modification. The lack of temporal and spatial control over the oxidation and deposition processes, however, limits possible applications of this diverse class of natural compounds. Autoxidation and deposition of phenolic compounds are uncontrollably triggered by basic $\mathrm{pH}$ and oxygen. In this project, inspired by the properties of natural antioxidants to scavenge free radicals and reactive oxygen species (ROS), we propose a method to effectively control the autoxidation and deposition of plant phenolic compounds under basic conditions. We demonstrate that natural antioxidants can inhibit autoxidation of plant polyphenols in basic $\mathrm{pH}$ in dark

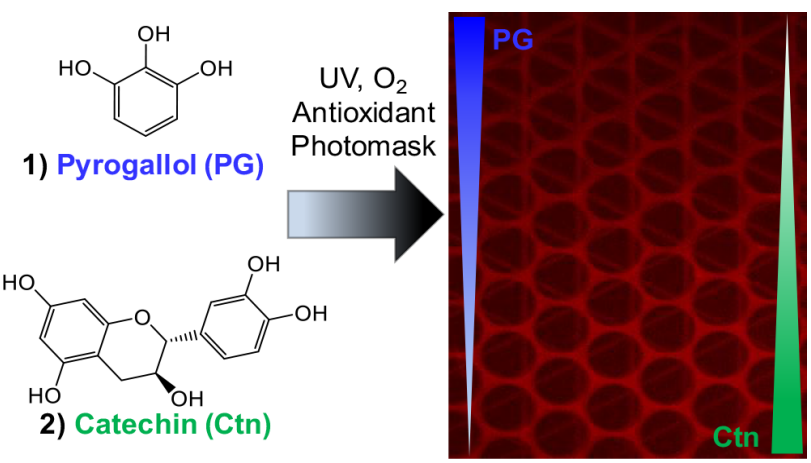
environment. However, UV irradiation of these solutions leads to on demand temporal and spatial polymerization and deposition of polyphenols. This bioinspired method was used to demonstrate the controlled polymerization, and micropatterning on flat surfaces and inside microfluidic channels opening the way to well defined $2 \mathrm{D}$ coatings based on natural polyphenols and introducing a new simple path to the fabrication of bioinspired functional materials, with potential applications in a wide range of fields.
\end{abstract}

\section{INTRODUCTION}

Plant derived polyphenols were recently introduced for particle and surface modification. ${ }^{1-3}$ Numerous applications in materials science have been already demonstrated including self healing materials, ${ }^{4}$ surface modification of membranes, designing novel antioxidants, ${ }^{6,7}$ anticancer drugs, ${ }^{8}$ and bio adhesives, ${ }^{9}$ as well as development of drug delivery systems, ${ }^{10}$ antibacterial coatings and surfaces with reduced biofouling, ${ }^{11}$ energy storage devices, ${ }^{12}$ thin film ${ }^{13-15}$ and bulk ${ }^{16}$ hybrid functional materials, and immobilization of enzymes. ${ }^{17,18}$

Phenolic compounds, similar to polycatecholamines such as dopamine (DA), can undergo autoxidation process in the presence of oxidizing agents, ${ }^{10}$ high redox potential enzymes which are suitable for use in oxidative processes, ${ }^{19}$ or in alkaline $\mathrm{pH}$ condition. ${ }^{20,21}$ However, because of the rapid response of phenolic compounds to oxidation, there is a limited control over the kinetics of the oxidation process and spatiotemporal control over the deposition of polyphenols, which leads to the formation of weak and inhomogeneous coatings. ${ }^{22}$ Formation and deposition of colloidal particles and aggregates have been observed in coatings made of catechol and pyrogallol containing compounds. ${ }^{22-26}$ Such colloidal structures on the surface, which cannot be easily avoided in the solution, have been considered as a source of instability and weakness of the anchoring layer and adlayer. ${ }^{22}$ Several research groups tried to make more homogeneous nanocoatings from polyphenols and DA by using new cyclic catechols, ${ }^{22}$ norepinephrine (a small molecule catecholamine), ${ }^{27}$ controlling the self polymerization process of DA by using the $\mathrm{CuSO}_{4} / \mathrm{H}_{2} \mathrm{O}_{2}$ system, ${ }^{28}$ and sonication of pDA coated substrate. ${ }^{29}$

Uncontrolled autoxidation and subsequent cross linking, polymerization, and complexation of catechol and pyrogallol moieties of plant phenolic compounds are dependent on multiple factors, such as $\mathrm{pH}$, buffer strength, concentration, and type of oxidant or enzymes. ${ }^{30}$ Many research groups tried to overcome uncontrolled autoxidation of pyrogallol and 
catechol containing compounds. Qiu et al. ${ }^{31}$ showed that enzyme induced deposition of tea catechin and chitosan depends on laccase enzyme concentration. Lee et al. ${ }^{32}$ used sodium periodate, horseradish peroxidase, and mushroom tyrosinase as oxidizing reagents to prepare 3,4 dihydroxyphe nylalanine modified (DOPA modified) polyethylene glycol (PEG). They observed that the amount of time required for gelation of aqueous PEG-DOPA solution was dependent on type and concentration of oxidizing agents. Ejima et al. ${ }^{33}$ showed that multivalent complexation of polyphenols and $\mathrm{Fe}(\mathrm{III})$ ions and film formation are directed by $\mathrm{pH}$ value of the solution. Barrett et $\mathrm{al}^{20}$ investigated the effect of $\mathrm{pH}$ on polymerization and deposition of a variety of plant phenolic compounds to find the optimum $\mathrm{pH}$ condition for nanocoating deposition. Hong et al. ${ }^{27}$ used norepinephrine, a small molecule catecholamine, in combination with dopamine to achieve a well controlled coating morphology. Formation of 3,4 dihydroxybenzaldehyde norepinephrine intermediates is con sidered as the main mechanism of the controlled oxidation process, leading to formation of a highly smooth nanocoating.

To form multifunctional coatings on the surface, $\mathrm{we}^{34-36}$ and other groups ${ }^{37-39}$ used UV light to induce oxidation of catechol and pyrogallol contacting compounds which enabled the control of the kinetics of the polymerization and deposition of these compounds spatiotemporally.

Several research groups ${ }^{2,11,20,39}$ including ours ${ }^{35}$ showed that spontaneous oxidation of plant phenolic compounds can be inhibited by using acidic $\mathrm{pH}$ conditions. However, this method may not be applicable to all phenolic compounds because of low solubility of some phenolic compounds at low $\mathrm{pH} .{ }^{20}$ Oxidation intermediates of phenolic compounds such as quinones are more stable at mild acidic condition $(\mathrm{pH}$ 5.76.7), decreasing the rate of cross linking, polymerization, and metal ion complexation. ${ }^{40}$ Cross linking of catechol or pyrogallol containing molecules by nucleophiles ${ }^{41}$ or their binding to tissues and proteins ${ }^{30}$ can be inhibited under acidic conditions.

Therefore, there is a clear need to develop a new strategy to control polymerization and cross linking of plant phenolic compounds under basic $\mathrm{pH}$. It is generally accepted that reactive oxygen species (ROS) can oxidize catechol and gallol moieties in plant derived phenolics to generate reactive species such as phenolate ion, phenolic radicals, semiquinone, and quinone. $^{42-44}$ Active quinone groups undergo phenolic coupling or react with nucleophiles such as thiols and amines. ${ }^{45}$ In our previous reports ${ }^{35,36}$ we investigated the role of reactive oxygen species [ROS, singlet oxygen $\left({ }^{1} \mathrm{O}_{2}\right)$, superoxide radical $\left(\mathrm{O}_{2}{ }^{--}\right)$and hydroxyl radical $\left.\left({ }^{\bullet} \mathrm{OH}\right)\right]$ in UV induced oxidation and polymerization of dopamine ${ }^{36}$ and plant phenolic compounds ${ }^{35}$ using spectroscopic and electrochemical meth ods. In human cells many antioxidants, such as sodium ascorbate (SA), uric acid (UA), and glutathione (GSH), can directly react with ROS or free radical intermediates induced by ROS (Scheme S1) and terminate the ROS induced damage. ${ }^{46}$ Recently, inspired by the ROS scavenging ability of sodium ascorbate (vitamin C) in the human body, we demonstrated that dopamine polymerization in basic solution can be inhibited by sodium ascorbate that reduces reactive dopamine quinone and delays dopamine polymerization. ${ }^{34}$ Several research groups discussed the mechanism of action of the natural antioxidant compounds including SA, ${ }^{47} \mathrm{UA},{ }^{48}$ and $\mathrm{GSH}^{49} \mathrm{SA}$ changes to the ascorbate radical by donating an electron to lipid radicals to terminate the lipid peroxidation chain reaction. ${ }^{50}$ The pairs of ascorbate radicals react rapidly to produce one molecule of ascorbate and one molecule of dehydroascorbate. ${ }^{50}$ Uric acid and glutathione are physiological natural antioxidants. Uric acid is the most abundant aqueous antioxidant found in humans. ${ }^{51}$ It contributes to as much as two thirds of all free radical scavenging activities in the plasma. ${ }^{51}$ The oxygen radical scavenging activity of glutathione directly expedites the ROS neutralization and the repair of ROS induced damage. The presence of the sulfhydryl group in glutathione allows it to serve as an antioxidant. ${ }^{50}$

In this work, inspired by the intrinsic function of natural antioxidants to scavenge ROS in plants, animals, and human cells, we developed a method to control the polymerization and surface functionalization by plant derived phenolic compounds in alkaline conditions ( $\mathrm{pH}$ 8.0). The method combines the effect of polymerization inhibition by antioxidants with the ability of UV light to trigger the polymerization of phenolic compounds, enabling spatiotemporal control of the polymer ization and deposition of various plant derived phenolic compounds. ${ }^{35}$ It should be noted that phenolic compounds are antioxidants by themselves, which require a stronger reducing agent to inhibit their oxidation at basic $\mathrm{pH}$. Interestingly, this method leads to more homogeneous polyphenolic films deposited on the surface. UV induced oxidation and polymerization of pyrogallol (PG), gallic acid (GA), pyrocatechol (Ctl), epigallocatechin gallate (EGCG), tannic acid (TA), catechin (Ctn), hydroxyhydroquinone (HHQ), caffeic acid (CA), and morin, some of the most common plant derived phenolic compounds (Figure 1), in the presence of sodium ascorbate (SA), glutathione (GSH), and uric acid (UA) as natural antioxidants were studied.

\section{EXPERIMENTAL SECTION}

Materials. Pyrogallol (PG), gallic acid (GA), pyrocatechol (Ctl), epigallocatechin gallate (EGCG), tannic acid (TA), catechin (Ctn), hydroxyhydroquinone (HHQ), caffeic acid (CA), and morin were purchased from Sigma Aldrich. L Ascorbic acid sodium salt and uric acid were purchased from Alfa Aesar. Glutathione (reduced) was purchased from Amresco (Germany). Silver nitrate, Rhodamine 110 chloride, and all the other chemicals were purchased from Sigma Aldrich and used without further purification. 2 Hydroxyethyl methacrylate (HEMA), ethylene dimethacrylate (EDMA) were purchased from Sigma Aldrich. Nexterion B glass slides obtained from Schott AG and silicon wafers (CZ Si wafer 4 in.) from MicroChem $\mathrm{GmbH}$ were used. Glass slides and silicon wafer substrates were cleaned by sonication in deionized (DI) water, 2 propanol, and $0.1 \mathrm{M} \mathrm{HCl}$ for $5 \mathrm{~min}$, washed with DI water, and dried with nitrogen gas. Flexible UV transparent fused silica capillary tubing (TSU100375 model) was purchased from Polymicro Technologies. High purity DI water with a resistivity of $18.2 \mathrm{M} \Omega \mathrm{cm}$ was obtained from an in line Millipore water purification system. Acetone and the other solvents were obtained from Merck KGaA. Phosphate buffers were made at $5 \mathrm{mmol} / \mathrm{L}$ concentration at $\mathrm{pH}$ 8.0. The final $\mathrm{pH}$ value adjusted by using a METTLER TOLEDO digital $\mathrm{pH}$ meter.

UV Irradiation of Phenolic Solutions. An OAI model 30 deep UV collimated light source (San Jose, CA) fitted with a $500 \mathrm{~W} \mathrm{HgXe}$ lamp was used for UV irradiation. The lamp was calibrated to $6 \mathrm{~mW} /$ $\mathrm{cm}^{2}$ at $260 \mathrm{~nm}$ with the OAI $306 \mathrm{UV}$ power meter. Reaction solutions were transferred into a quartz cuvette and irradiated under UV lamp for $2 \mathrm{~h}$ (unless stated otherwise) at room temperature, ambient atmosphere.

UV-Vis absorption experiments. A $2 \mathrm{~mL}$ phenolic compound solution $(0.2 \mathrm{mg} / \mathrm{mL}$, unless stated otherwise $)$ in the phosphate buffers at $\mathrm{pH} 8.0(5 \mathrm{mmol} / \mathrm{L})$ was stored in dark environment (dark samples) and irradiated under UV (UV samples) in quartz cuvettes for the desired time. The UV-vis absorbance $(230-700 \mathrm{~nm})$ of the dark 
(A)

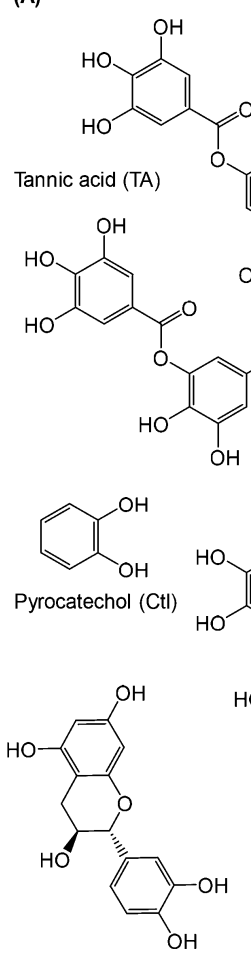

Catechin (Ctn)

(B)

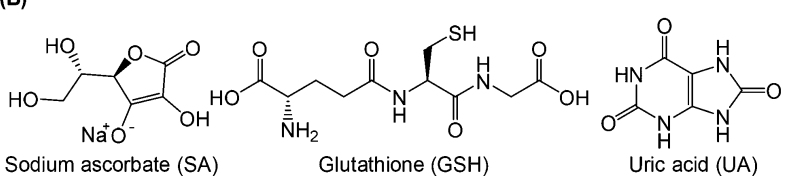

Figure 1. Chemical structures of plant derived natural phenolic compounds commonly used for preparing functional polyphenolic coatings (A) and structures of physiologically important antioxidants (B).

and UV samples was measured at different time points $(0,5,15,30$, 45, 60, 90, and $120 \mathrm{~min}$; buffer as the reference) using a Lambda 35 UV-vis spectrometer (PerkinElmer). For sample stored for a longer time, $2 \mathrm{~mL}$ of the solution was added into a quartz cuvette and sealed for each time point. UV-vis spectra were measured after 6, 12, 24, 48, and $72 \mathrm{~h}$. Morin solution was prepared at $0.05 \mathrm{mg} / \mathrm{mL}$ in the phosphate buffer at $\mathrm{pH} 8.0(5 \mathrm{mmol} / \mathrm{L})$. For samples contacting antioxidants, the desired amount of the antioxidant was added to the buffer solution, and then the phenol was added to this solution. Because of the solubility issue of UA, PG solutions containing UA were prepared at $0.1 \mathrm{mg} / \mathrm{mL}$ in a $2.5 \mathrm{mmol} / \mathrm{L}$ phosphate buffer at $\mathrm{pH}$ 8.0 .

For on demand polymerization experiments, PG solution $(0.2 \mathrm{mg} /$ $\mathrm{mL}$, phosphate buffer $\mathrm{pH} 8.0,5 \mathrm{mmol} / \mathrm{L}$, with $\mathrm{GSH} / \mathrm{PG}$ molar ratio of $0.8: 1$ or without antioxidant) was kept in the dark for $30,60,90,120$, 150 , and $180 \mathrm{~min}$ before recording the solution's UV-vis absorption spectra. After UV irradiation for $5 \mathrm{~min}$ at 30, 60, 90, and $120 \mathrm{~min}$ the solution was placed in the dark, and the solution's UV-vis spectrum was recorded every $30 \mathrm{~min}$ for $3 \mathrm{~h}$. Similar experiments were done on PG solutions with $5 \mathrm{~min}$ of UV irradiation at different time points (30, $60,90,120$, and $150 \mathrm{~min}$ ), and the UV-vis spectra of the solutions were recorded every $30 \mathrm{~min}$. In another set of experiments the solution was stored in dark and continuously irradiated with UV light at different time points, and the UV-vis spectra were recorded every 30 $\min$ for $3 \mathrm{~h}$

Electrochemistry. All electrochemical measurements were per formed with a Reference 600 potentiostat (Gamry Instruments, Warminster, PA) in a three electrode cell with a coiled Pt wire as the counter electrode, and a $\mathrm{Ag} / \mathrm{AgCl}(3 \mathrm{~mol} / \mathrm{L} \mathrm{KCl})$ reference electrode. The working electrodes were a specifically activated glassy carbon disk electrode ( $3 \mathrm{~mm}$ in diameter) prepared as described in the literature. ${ }^{52}$ The electrodes were polished with $0.3 \mu \mathrm{m}$ alumina slurry and activated before each measurement. The phenolic compounds $(1.58 \mathrm{mmol} / \mathrm{L})$ were dissolved in phosphate buffer $(5 \mathrm{mmol} / \mathrm{L})$ at $\mathrm{pH} 8.0$ (vide supra). A neoLab UV inspection lamp Type 6 with $14 \mathrm{~mW} / \mathrm{cm}^{2}$ was used for UV irradiation and positioned at a distance of $0.5 \mathrm{~cm}$ from a quartz cuvette containing the solution of the phenolic compound. The differential pulse voltammetry (DPV) was measured from -0.2 to 0.8 $\mathrm{V}$ with $25 \mathrm{mV}$ pulse size, and the frequency is $5 \mathrm{~Hz}$. The low buffer concentration does not provide sufficient ionic strength for the DPV measurements. Therefore, $0.1 \mathrm{~mol} / \mathrm{L} \mathrm{KCl}$ was added to the solution before measurement.

Deposition of the Polyphenolic Layer on the Silicon Surface. Sodium chloride $(60 \mathrm{mmol} / \mathrm{L})$ was added to the phosphate buffers $(5$ $\mathrm{mmol} / \mathrm{L}$ ) in the deposition and micropatterning process to increase the ionic strength of the solution. Clean silicon substrates were immersed in buffered solutions of $0.2 \mathrm{mg} / \mathrm{mL}$ PG with SA (SA/PG molar ratio $0.4: 1$ ) or without SA for $30 \mathrm{~min}$ in dark room or under UV irradiation at room temperature. Modified samples were then rinsed thoroughly with DI water and ethanol and dried with nitrogen gas followed by a gentle scratch with one tip of a pair of tweezers. Similarly, a phenolic layer was deposited on the surface of silicon by immersing the silicon surface in a $0.2 \mathrm{mg} / \mathrm{mL}(0.05 \mathrm{mg} / \mathrm{mL}$ for morin) phenolic solution of Ctl, EGCG, TA, Ctn, HHQ and morin containing SA at SA/phenol molar ratios of $0.15,0.70,0.85,0.4$, 1.50 , and $0.30: 1$, respectively, and the samples were stored overnight in dark. The same conditions were used to make a phenolic layer on the silicon surface under UV irradiation for $30 \mathrm{~min}$.

Photopatterning of Phenolics. For patterning on the substrate, a photomask was fixed on top of the poly(HEMA EDMA) substrate using a setup described in our previous report. ${ }^{35}$ Details of nanoporous poly(HEMA EDMA) preparation could be found in our report. ${ }^{53} \mathrm{~A}$ $0.2 \mathrm{mg} / \mathrm{mL}$ phenolic solution of PG, GA, Ctl, EGCG, TA, Ctn, and HHQ containing SA at SA/phenol molar ratios of 0.4, 0.1, 0.15, 0.70, $0.85,0.4$, and $1.50: 1$, respectively, was filled in between the photomask and the substrate, and the sample was UV irradiated for $10 \mathrm{~min}$. Then, the photomask was removed, and the sample was rinsed with DI water and ethanol and dried with $\mathrm{N}_{2}$. For the secondary modification by $\mathrm{AgNO}_{3}$, the patterned substrates were immersed into a $10 \mathrm{mmol} / \mathrm{L}$ $\mathrm{AgNO}_{3}$ aqueous solution overnight, followed by washing with water and drying with $\mathrm{N}_{2}$. For the secondary modification by Rhodamine 110 , the patterned substrates were immersed in an ethanol/phosphate buffer ( $\mathrm{pH} 8.0,5 \mathrm{mmol} / \mathrm{L}$ ) 1:1 mixture containing $0.1 \mathrm{~g} / \mathrm{mL}$ of dye overnight; then, the substrate was carefully washed with deionized water and ethanol and dried with $\mathrm{N}_{2}$.

Sequential Patterning of Polyphenols. For the preparation of the "KIT" pattern of three different polyphenols, first, the left part of the KIT logo was deposited on the surface of poly(HEMA EDMA) by irradiating the PG solution $(0.2 \mathrm{mg} / \mathrm{mL}$, phosphate buffer $5 \mathrm{mmol} / \mathrm{L}$, $\mathrm{pH}$ 8.0) containing SA/PG 0.4:1 molar ratio through a photomask, followed by washing the substrate with water and ethanol and secondary modification with Rhodamine 110 by incubation in dye solution overnight as explained before. Then, the " $\mathrm{K}$ " and " $\mathrm{T}$ " letters, the middle and right part of the logo, were deposited on the surface by UV irradiation of Ctn and HHQ solutions $(0.2 \mathrm{mg} / \mathrm{mL}$, phosphate buffer $5 \mathrm{mmol} / \mathrm{L}$, pH 8.0) containing SA/phenol 0.4, 1.5:1 molar ratio through the photomask for $10 \mathrm{~min}$, respectively, followed by washing after each step. We used three different filter sets [filter set 1 (DsRED), excitation filter $510-560 \mathrm{~nm}$, emission filter $590-650 \mathrm{~nm}$; filter set 2 (GFP plants), excitation filter $450-490 \mathrm{~nm}$, emission filter $500-550 \mathrm{~nm}$; filter set 3 (CFP), excitation filter $426-446 \mathrm{~nm}$, emission filter $460-500 \mathrm{~nm}$ ] to visualize each phenolic pattern. The "KIT" pattern was modified with silver particles by immersing it in a silver nitrate $10 \mathrm{mmol} / \mathrm{L}$ aqueous solution overnight.

An overlaid pattern of polyphenols was made on the poly(HEMA EDMA) surface first by irradiation of PG solution $(0.2 \mathrm{mg} / \mathrm{mL}$, phosphate buffer $5 \mathrm{mmol} / \mathrm{L}$, pH 8.0) containing SA with SA/PG 0.4:1 molar ratio and subsequent secondary modification with Rhodamine 
(A)
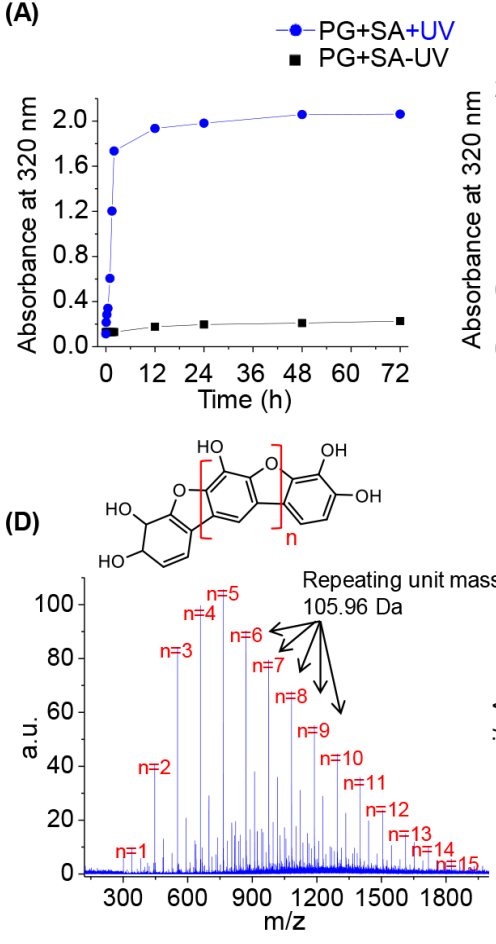

(B)

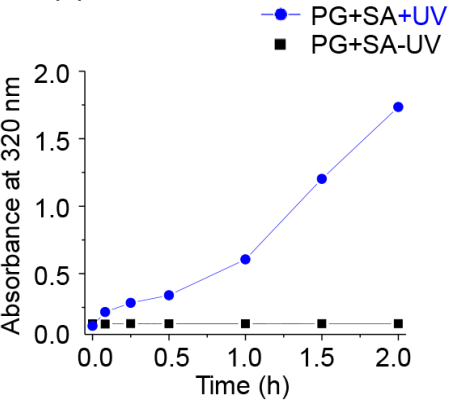

(C)

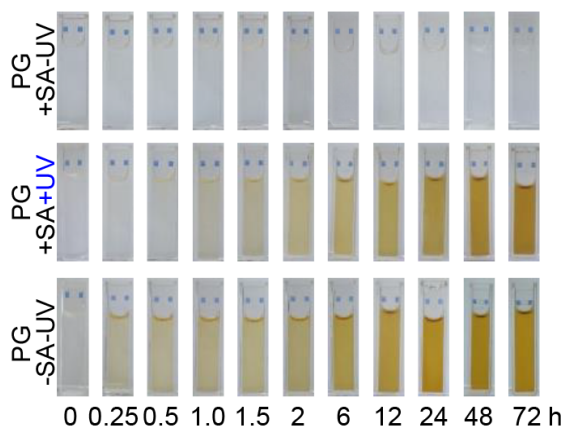

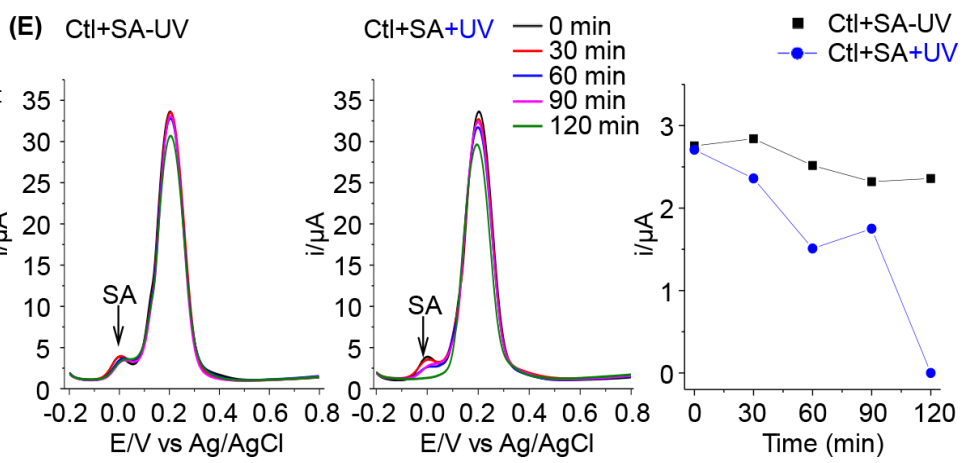

Figure 2. Effect of natural antioxidants on PG oxidation in dark and under UV irradiation. The graphs show UV absorbance of PG solutions (0.2 $\mathrm{mg} / \mathrm{mL}$ of PG in phosphate buffer, $5 \mathrm{mmol} / \mathrm{L}, \mathrm{pH} 8.0$ ) at $320 \mathrm{~nm}$. (A) UV absorbance of PG solution over $72 \mathrm{~h}$ stored in the dark or after $2 \mathrm{~h}$ of UV irradiation. (B) UV absorbance at $320 \mathrm{~nm}$ of PG solution with SA (SA/PG 1.2:1 molar ratio) and without SA in dark and under UV irradiation. (C) Corresponding photographs of each sample. (D) ESI MS spectra (positive mode) of the PG solution $(0.2 \mathrm{mg} / \mathrm{mL}$ of PG in phosphate buffer, 5 $\mathrm{mmol} / \mathrm{L}, \mathrm{pH}$ 8.0; 1.2:1, SA/PG molar ratio) after UV irradiation for $2 \mathrm{~h}$ indicates generation of higher molecular weight species with a repeating unit of 105.96 Da. (E) Differential pulse voltammetry (DPV) of Ctl solution $(0.2 \mathrm{mg} / \mathrm{mL}$ of Ctl in phosphate buffer, $5 \mathrm{mmol} / \mathrm{L}, \mathrm{pH} 8.0 ; 0.6: 1, \mathrm{SA} / \mathrm{Ctl}$ molar ratio) with $\mathrm{KCl}(0.1 \mathrm{~mol} / \mathrm{L})$ at activated glassy carbon disk electrodes stored in dark (left) or under UV irradiation (middle) for $2 \mathrm{~h}$. Peak currents for SA oxidation (at $0 \mathrm{~V}$ vs $\mathrm{Ag} / \mathrm{AgCl} \mathrm{KCl} 3 \mathrm{~mol} / \mathrm{L}$ ) vs time in dark and under $\mathrm{UV}$ irradiation (right).

110 as described before. The second pattern was formed by irradiation of Ctn solution $(0.2 \mathrm{mg} / \mathrm{mL}$, phosphate buffer $5 \mathrm{mmol} / \mathrm{L}$, $\mathrm{pH} 8.0)$ containing SA with SA/PG 0.4:1 molar ratio followed by washing with water and ethanol after each step. We used two different filter sets [filter set 4 (TexasRed), excitation filter 522-602 nm, emission filter $584-664 \mathrm{~nm}$; filter set 5 (DAPI BP), excitation filter $327-427 \mathrm{~nm}$, emission filter 387-507 nm] to visualize each phenolic pattern.

Gradient Pattern. For a gradient of the polyphenolic pattern, poly(HEMA EDMA) modified substrate was fed into the patterning setup described before, and filled with PG solution $(0.2 \mathrm{mg} / \mathrm{mL}$, phosphate buffer $5 \mathrm{mmol} / \mathrm{L}$, $\mathrm{pH}$ 8.0) containing SA with SA/PG 0.4:1 molar ratio. A black cardboard cover was used to cover the photomask. For a gradient pattern of polyphenol, the cardboard was pulled off the photomask gradually within $5 \mathrm{~min}$. Gradient patterns of polyphenols were modified with silver particles as described before. For overlaid gradient patterns, after deposition of the first pattern (triangle) by 5 min of UV irradiation of PG solution through a photomask and modification with Rhodamine as describe before, the second gradient pattern (hexagonal) was deposited on the same substrate by UV irradiation of Ctn solution through a photomask for another $5 \mathrm{~min}$ as describe before. The black cardboard was pulled off in the opposite direction (from right to left for PG and from left to right for Ctn) to make a contrast of patterns on two different sides. The substrate was immersed overnight in $10 \mathrm{mmol} / \mathrm{L}$ aqueous silver nitrate solution or Rhodamine dye solution for modification with silver or Rhodamine.

Patterning Inside a Capillary. First, capillaries were modified with porous poly(HEMA EDMA) according to our previous report. ${ }^{35}$ Briefly, capillaries were filled with a sodium hydroxide solution (1 $\mathrm{mol} / \mathrm{L}$ ) for $1 \mathrm{~h}$, followed by rinsing with DI water, then filling with an $\mathrm{HCl}$ solution $(1 \mathrm{~mol} / \mathrm{L})$ for $30 \mathrm{~min}$, then washing with DI water and drying with pumping air inside the capillary. The activated glass surface was functionalized with 20 vol \% 3 (trimethoxysilyl)propyl meth acrylate in ethanol for $30 \mathrm{~min}$ followed by washing with ethanol. The polymerization mixture (HEMA 24 wt \%, EDMA 16 wt \%, 1 decanol 45.5 wt \%, cyclohexanol 14.5 wt \%, 2,2 dimethoxy 2 phenyl acetophenone $1 \mathrm{wt} \%$ with respect to monomers) was injected into the modified capillary using a syringe. The capillary filled with the polymerization mixture was placed under the UV lamp and irradiated with UV light for $15 \mathrm{~min}$ (the lamp was calibrated to $6 \mathrm{~mW} / \mathrm{cm}^{2}$ at $260 \mathrm{~nm}$ with the OAI $306 \mathrm{UV}$ power meter) followed by washing with ethanol. A porous polymer was formed inside the capillary. For a polyphenolic pattern inside the capillary, PG solution was injected into the capillary using a syringe. The capillary filled with the PG $(0.2 \mathrm{mg} /$ $\mathrm{mL}$, phosphate buffer $5 \mathrm{mmol} / \mathrm{L}, \mathrm{pH} 8.0$ ) containing SA with SA/PG 0.4:1 molar ratio was placed under a photomask and irradiated with UV light $\left(6 \mathrm{~mW} / \mathrm{cm}^{2}\right.$ at $\left.260 \mathrm{~nm}\right)$ for $10 \mathrm{~min}$ followed by washing with DI water and acetone. For secondary modification with silver particles and fluorescent dye, an aqueous solution of silver nitrate or Rhodamine 110 chloride solution described before was injected into the capillaries and reacted overnight, followed by washing with DI water and acetone and drying with air.

Characterization. UV-vis spectroscopy was performed with a Lambda 35 UV-vis spectrometer (PerkinElmer). The bright field and fluorescence images were taken using a Leica DFC360 microscope and Keyence BZ 9000 microscope. Image J software was used to measure gray value. Mass analysis was performed using an ESI MS (Bruker ESI TOF in INT, KIT) instrument in positive mode. Atomic force microscopy (AFM) was performed on a Dimension Icon AFM (Bruker) in standard tapping mode in air, INT, KIT. Cantilevers used were of type HQ:NSC15/AI BS (MikroMasch) with a nominal force constant of $40 \mathrm{~N} / \mathrm{m}$ and a resonance frequency of $325 \mathrm{kHz}$. The distributions of phenolic mass fragments on the surface were 
(A)

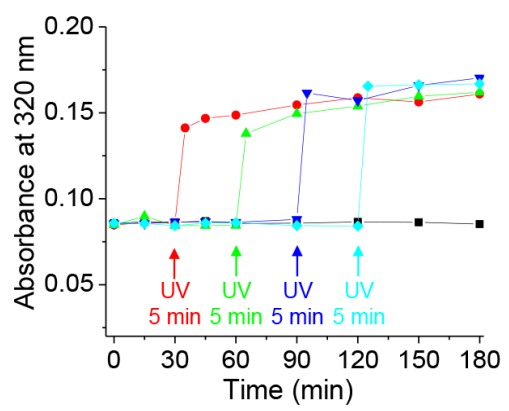

(B)

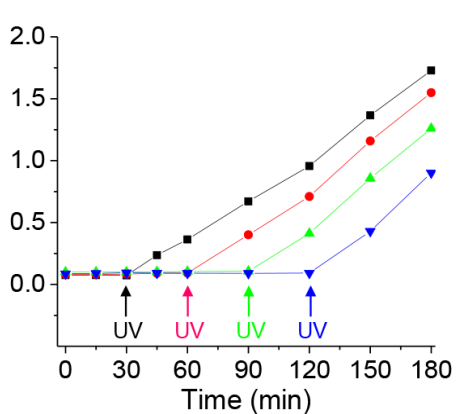

(C)

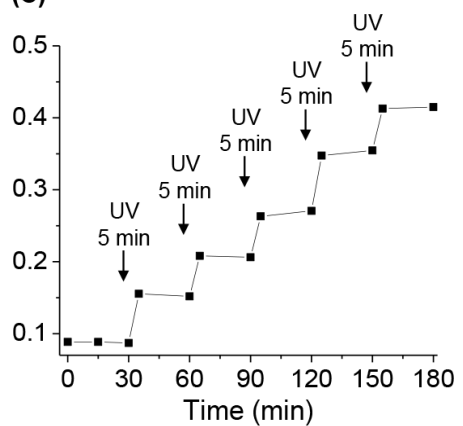

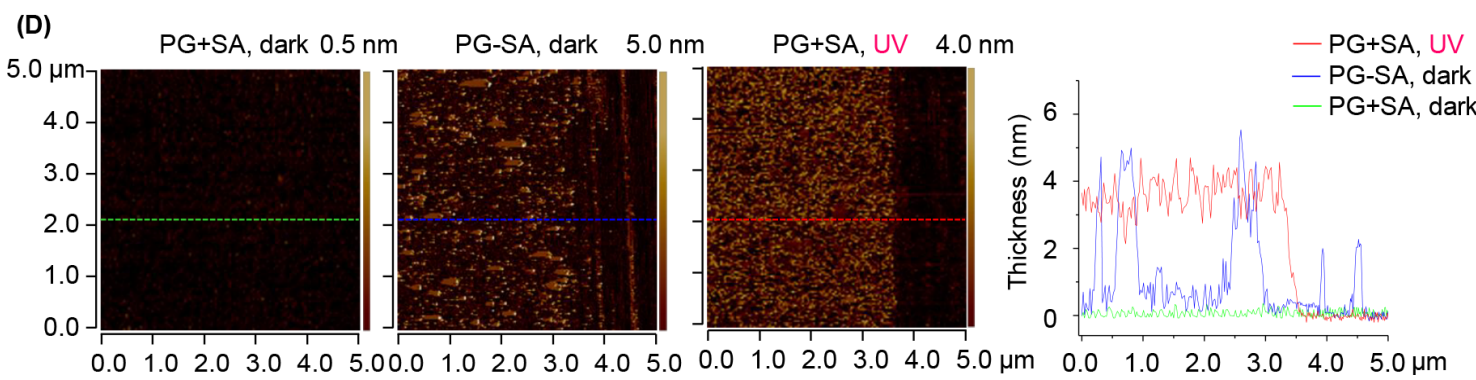

Figure 3. On demand initiation and termination of PG polymerization in the presence of GSH (GSH/PG molar ratio of $0.8: 1$, phosphate buffer 5 $\mathrm{mmol} / \mathrm{L}, \mathrm{pH} \mathrm{8.0)}$. (A) By UV irradiation of a different GSH PG solution at different time intervals, PG polymerization can be initiated, and by removing UV light, the polymerization reaction stops again. (B) As long as dormant GSH PG solution is irradiated with UV light, polymerization initiates and proceeds. (C) Polymerization of PG proceeds stepwise by irradiating the GSH PG solution for 5 min at different time intervals. (D) AFM on silicon surfaces exposed to PG solution at pH $8.0(5 \mathrm{mmol} / \mathrm{L}$ ) with SA in dark (left), without SA in dark (middle), and with SA after 30 min of UV irradiation, gently scratched by tweezers. Surface topographies measured along the dashed lines (graph).

investigated with time of flight secondary ion mass spectrometry (ToF SIMS) (ION TOF Inc.), IFG, KIT. XPS measurements were performed using a KAlpha+ XPS spectrometer (ThermoFisher Scientific, East Grinstead, UK), IAM, KIT. Data acquisition and processing using the Thermo Advantage software are described elsewhere. ${ }^{54}$ All coatings were analyzed using a microfocused, monochromated $\mathrm{Al} \mathrm{K} \alpha \mathrm{X}$ ray source $(400 \mu \mathrm{m}$ spot size). The $\mathrm{K}$ Alpha charge compensation system was employed during analysis, using electrons of $8 \mathrm{eV}$ energy, and low energy argon ions to prevent any localized charge build up. The spectra were fitted with one or more Voigt profiles (BE uncertainty: $+0.2 \mathrm{eV}$ ), and Scofield sensitivity factors were applied for quantification. ${ }^{55}$ All spectra were referenced to the $\mathrm{C} 1 \mathrm{~s}$ peak $(\mathrm{C}-\mathrm{C}, \mathrm{C}-\mathrm{H})$ at $285.0 \mathrm{eV}$ binding energy controlled by means of the well known photoelectron peaks of metallic $\mathrm{Cu}, \mathrm{Ag}$, and $\mathrm{Au}$, respectively. The $\mathrm{K}$ alpha+ snapmap option was used to image an area of $2 \times 2 \mathrm{~mm}$ with an X ray spot of $200 \mu \mathrm{m}$ (5 iterations were run to reach a better statistic).

\section{RESULTS AND DISCUSSION}

To investigate our hypothesis that natural antioxidants can inhibit polymerization and deposition of natural phenolic compounds under basic conditions we, first, performed UV-vis spectroscopic and differential pulse voltammetric analysis of plant phenolic solutions at $\mathrm{pH} 8.0$ in the presence or absence of antioxidants both in dark and under UV irradiation. UV-vis spectra of PG solution $(0.2 \mathrm{mg} / \mathrm{mL})$ with different SA/PG molar ratios from 0:1 up to 1.2:1 were measured in the dark and under UV irradiation (Figure S1). UV absorbance at 320 $\mathrm{nm}$ (at $380 \mathrm{~nm}$ for TA) was selected as an indication of quinone formation in the solution to monitor oxidation and polymerization of the phenolic compounds. The results demonstrate that oxidation of PG begins immediately at $\mathrm{pH}$ 8.0 either in the dark or under UV irradiation (Figure 2). By increasing the amount of SA present in the solution, however, the rate of oxidation of PG decreases for both samples (Figure
$\mathrm{S} 2)$. At the molar ratio as high as 1.2:1 (SA/PG), the oxidation of PG in the dark environment is completely ceased for at least $72 \mathrm{~h}$ (Figure 2A), while UV irradiation of the sample at $260 \mathrm{~nm}$ $\left(6 \mathrm{~mW} / \mathrm{cm}^{2}\right)$ triggered the oxidation and polymerization process, resulting in a 15 fold increase of UV absorbance at $320 \mathrm{~nm}$ (from 0.11 to 1.73 ) already after $2 \mathrm{~h}$ (Figure 2B). The color of the PG solution stored in dark did not change in the presence of SA/PG 1.2:1 molar ratio. However, color of the same PG solution irradiated with UV light for $2 \mathrm{~h}$ and color of the PG solution without SA changed over time (Figure 2C). Electrospray ionization time of flight mass spectrometry (ESI TOF) of the pyrogallol solution ( $\mathrm{pH} 8.0,5 \mathrm{mmol} / \mathrm{L}, \mathrm{SA} / \mathrm{PG}$ molar ratio of 1.2:1) indicated the generation of higher molecular weight species after UV irradiation of the solution for $2 \mathrm{~h}$ (Figure 2D, and Figure S3). A repeating unit with the mass of $105.96 \mathrm{Da}$ was observed in the mass spectra which corresponds to a structure shown in Figure 2D. ${ }^{35}$

To show that antioxidant controlled oxidation of plant phenolic compounds is a general concept, we used glutathione (GSH) and uric acid (UA) to study the kinetics of PG oxidation at $\mathrm{pH} 8.0$ both in dark and under UV irradiation by UV-vis spectroscopy (Figures S4-S7). Presence of glutathione at GSH/PG 0.8:1 molar ratio could inhibit the oxidation of PG for $2 \mathrm{~h}$ (Figure S4). However, $2 \mathrm{~h}$ of UV irradiation of the same sample caused a 13 fold increase in UV absorbance at $320 \mathrm{~nm}$ (from 0.09 to 1.22) (Figure S5). A similar effect was observed by using uric acid (UA) as a reducing agent (Figure S6). At UA/PG 1.40:1 molar ratio, oxidation of PG was significantly slowed down in dark, while UV irradiation triggered the oxidation of PG, and the UV absorbance of the PG solution increased 6 fold (from 0.55 to 3.45 ) after UV irradiation (Figure S7). 
To show that natural antioxidants can be used to effectively control the rate of oxidation of other natural plant derived phenolic compounds, we investigated a library of plant phenolic compounds including gallic acid (GA), pyrocatechol (Ctl), epigallocatechin gallate (EGCG), tannic acid (TA), catechin (Ctn), hydroxyhydroquinone (HHQ), caffeic acid (CA), and morin. Basic solutions of the phenolic compounds $(\mathrm{pH} 8.0,5$ $\mathrm{mmol} / \mathrm{L}$ phosphate buffer) containing different concentrations of SA were analyzed by UV-vis spectroscopy after UV irradiation for $2 \mathrm{~h}$ or storage in the dark. Addition of SA to the solutions at $\mathrm{pH} 8.0$ decreased the rate of oxidation both in dark and under UV irradiation (Figures S8-S15). At SA/ phenol molar ratios listed in Table S1, oxidation of phenolic compounds is completely stopped for at least $2 \mathrm{~h}$ in the dark. However, UV irradiation at these molar ratios could still trigger the oxidation followed by an increase in UV absorbance (Figures S8-S15). The $\mathrm{pH}$ value of the solutions after addition of antioxidants did not change confirming that the inhibition ability of the antioxidants was a result of their ROS scavenging and reducing properties.

To confirm the inhibition effect of SA and the UV acceleration effect on the polymerization of plant phenolic compounds at $\mathrm{pH} 8.0$, we used electroanalytical methods to monitor the change in the SA oxidation peak response as a measure of the SA concentration. We used differential pulse voltammetry (DPV) with specifically activated glassy carbon electrodes to discriminate the respective signals of SA, Ctl, and CA in the SA-phenol mixtures. The SA oxidation in the SAphenol mixtures appears as a small prepeak or shoulder (at $0 \mathrm{~V}$ versus $\mathrm{Ag} / \mathrm{AgCl}$ ) of the larger $\mathrm{Ctl}$ or $\mathrm{CA}$ oxidation peak at 0.2 and $0.25 \mathrm{~V}$ versus $\mathrm{Ag} / \mathrm{AgCl}$, respectively (Figure 2E, Figures S16 and S17). Monitoring of SA by means of DPV demonstrates that the oxidation rates of SA increased after $\mathrm{UV}$ irradiation of the solution for $2 \mathrm{~h}$ (Figure $2 \mathrm{E}$ ). This phenomenon can be attributed to the formation of ROS from dissolved oxygen under UV irradiation, such as singlet oxygen $\left({ }^{1} \mathrm{O}_{2}\right)$, superoxide radicals $\left(\mathrm{O}_{2}{ }^{\bullet-}\right)$, or hydroxyl radicals $\left({ }^{\bullet} \mathrm{OH}\right)$. Under UV irradiation, the DPV results of CA display a new peak around $0.35 \mathrm{~V}$ versus $\mathrm{Ag} / \mathrm{AgCl}$ assigned to intermediates of the polymerization processes. In absence of SA, the peak for CA almost fully disappears within only $30 \mathrm{~min}$ of irradiation. In the presence of $\mathrm{SA}$, while polymerization intermediates are still detected, the CA peak only decreases moderately within $2 \mathrm{~h}$ (Figure S17). Overall, the consumption of SA in the SAphenol mixture and the fast consumption of phenols in the SA free solutions confirm that the SA functions as a reductant decreasing the rate of phenolic compound oxidation both in dark and under UV irradiation. Thus, phenolic compounds being natural antioxidants by themselves can be kept in their reduced form in the presence of stronger reducing agents such as SA.

UV-vis spectroscopy and electrochemistry results demon strate the ability of natural antioxidants to effectively inhibit the oxidation of plant phenolic compounds under basic conditions, while UV irradiation can be used for the temporal control of the oxidation and polymerization of polyphenolic compounds. To exemplify this possibility, we used a PG solution with the GSH additive (GSH/PG molar ratio of 0.8:1; $\mathrm{pH} 8.0$ ), which does not oxidize for $120 \mathrm{~min}$ (Figure 3A, Figure S18). However, irradiating the solution for $5 \mathrm{~min}$ at any moment triggered the oxidation of PG leading to an immediate increase in the UV absorbance at $320 \mathrm{~nm}$ from 0.08 to around 0.15 (Figure $3 \mathrm{~A})$. In this case, the polymerization of PG does not continue after the UV pulse. However, if the UV irradiation continues, oxidation, polymerization, and deposition of PG steadily continue further as indicated by a gradual increase in UV absorbance at $320 \mathrm{~nm}$ (Figure 3B). By repeating the UV irradiation for $5 \mathrm{~min}$ followed by storing in dark, it was possible to drive the oxidation process of PG to the desired stage at each particular time point for several times (Figure 3C), thereby making a stepwise control of the polymerization of PG possible in the presence of antioxidant. In a control experiment, the PG solution without GSH was irradiated with UV pulses for $5 \mathrm{~min}$ at 0,25 , and $55 \mathrm{~min}$, and the UV absorbance at $320 \mathrm{~nm}$ was measured. In the absence of antioxidant, continuous autox idation of PG was observed even after the UV pulses (Figure S19).

Precipitation or surface adsorption of insoluble higher molecular weight oligomers and adsorption of colloidal polymer particles, formed from self assembled polyphenol oligomers in solution, are the main mechanisms for the formation of coatings based on polyphenolic compounds. ${ }^{11} \mathrm{We}$ investigated the formation of polyphenolic layers and its homogeneity on a silicon wafer surface in the presence and absence of SA and with or without UV irradiation. AFM analysis demonstrated no coating on a surface treated with an SA/PG solution $(0.2 \mathrm{mg} / \mathrm{mL}, 5 \mathrm{mmol} / \mathrm{L}$ phosphate buffer, $\mathrm{pH}$ 8.0; SA/PG molar ratio $0.4: 1$ ) without UV irradiation. The same procedure with $30 \mathrm{~min}$ of UV irradiation resulted in the formation of a $4 \mathrm{~nm}$ thin coating with $0.98 \mathrm{~nm}$ roughness $\left(R_{\mathrm{q}}\right)$ (Figure 3D, Figure S20D). The polyphenolic layer formed on a silicon wafer in a PG solution without SA and with no UV light was $4 \mathrm{~nm}$ thick with $R_{\mathrm{q}}$ roughness $2.33 \mathrm{~nm}$ (Figure 3D, Figure S20B). The same protocol but with $30 \mathrm{~min}$ of UV irradiation results in a $5 \mathrm{~nm}$ coating being also highly inhomogeneous with $R_{\mathrm{q}}$ of $3.51 \mathrm{~nm}$ (Figure S20C). Thus, addition of SA into the PG solution resulted in the deposition of a more homogeneous polyphenolic layer, deposition of which could be temporally controlled by UV irradiation.

Roughness $\left(R_{\mathrm{q}}\right)$ of the phenolic nanocoating on the silicon surface was measured for samples without antioxidants stored in dark environment, and for UV irradiated samples containing SA. A lower value of $R_{\mathrm{q}}$ was observed in Ctl, EGCG, TA, and HHQ samples containing SA compared to the samples without SA (Figure S21). However, $R_{\mathrm{q}}$ of Ctn and morin remained almost constant for the samples with and without SA (Figure S21). Different roughnesses obtained with or without SA could be due to the difference in the kinetics of oxidation and deposition of each phenolic compound in the presence or absence of SA.

Through a change in the oxidation condition from dark to UV exposed environment, kinetics of the oxidation of phenolic compounds was accelerated as it was seen in UV-vis spectroscopy and electrochemistry analysis (Figures S1-S17). However, the overall oxidation behavior of the phenolic compounds (such as peak shapes and peak positions in UVvis and electrochemistry graphs) remained almost constant in dark and under UV. In our previous work, ${ }^{35}$ similar behavior was observed for different plant phenolic compounds at both acidic and basic solutions. Second, nanoparticle like aggregates were observed on the surface after coating deposition from phenolic compounds both in dark and under UV (Figures S20 and S21). Similar behavior of oxidation and deposition observed in UV-vis spectroscopy, electrochemistry, and AFM images suggests that similar radical intermediates formed during the oxidation process in dark and under UV irradiation. 
UV-vis spectroscopy, mass spectrometry, electrochemical analysis, and AFM results indicate that UV irradiation could accelerate polymerization and deposition of plant phenolic compounds, whereas SA is able to inhibit the oxidation of phenols either through direct scavenging of ROS in the solution or via reducing of quinone moieties of phenols. On one hand, ROS can increase consumption of SA, and on the other hand, ROS accelerates oxidation rate of phenols as was reported before. $^{34-36}$ The principle of antioxidant controlled oxidation of phenolic compounds in the presence of sodium ascorbate (SA) is depicted in Scheme 1.

Scheme 1. Schematic Illustrating Antioxidant Controlled Oxidation of Plant Phenolic Compounds

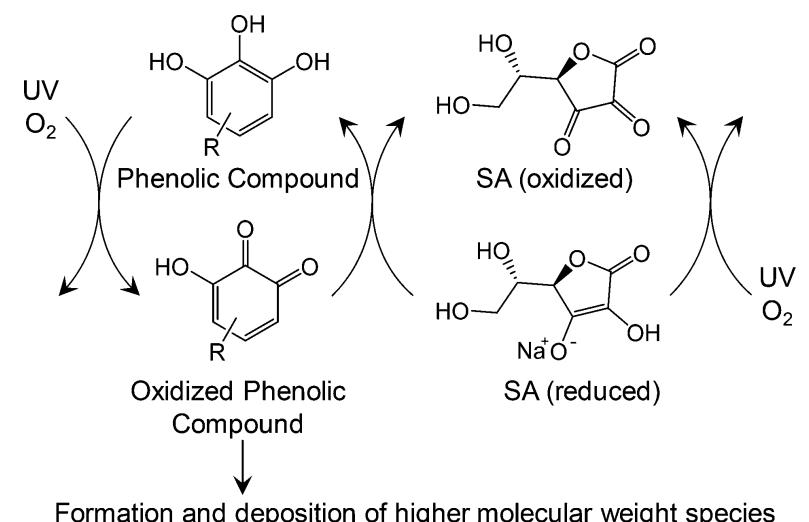

Light is uniquely suited for spatiotemporal control of reactions and surface functionalization. The ability to use UV light to trigger polymerization and deposition of structurally diverse natural phenolic compounds, under alkaline conditions where such polymerization and deposition is the fastest, opens endless opportunities for the formation of functional polyphenol patterning, copatterning of different phenolic compounds, and patterning of phenolics inside of closed systems nonaccessible to stamping methods.

For evaluation of the ability to pattern phenolic compounds, the poly( 2 hydroxyethyl methacrylate) co (ethylene dimetha crylate) (poly(HEMA EDMA)) surface was coated with a solution of PG $(0.2 \mathrm{mg} / \mathrm{mL}$, phosphate buffer $5 \mathrm{mmol} / \mathrm{L}$, $\mathrm{pH}$ 8.0, SA/PG molar ratio 0.4:1), which was covered with a quartz photomask, followed by 10 min of UV irradiation. The surface was then washed with deionized (DI) water and ethanol followed by drying with $\mathrm{N}_{2}$. For visualization of the patterns, the surface was immersed in silver nitrate aqueous solution (10 $\mathrm{mmol} / \mathrm{L})$ or Rhodamine 110 solution $(0.1 \mathrm{~g} / \mathrm{mL})$ overnight (Figure S22A).

With this method we were able to create patterns using various phenolic compounds with features down to $10 \mu \mathrm{m}$ (Figure S22A-F). We also evaluate the possibility to create multiple patterns by a sequential wetting of the same surface with different phenolic compound solutions and their UV irradiation for $10 \mathrm{~min}$ using a photomask. We used the autofluorescence characteristic of plant phenolic com pounds ${ }^{56,57}$ to image the patterns. The results (Figure 4) demonstrate clear patterns of different phenolic compounds, indicating the lack of deposition of phenolics in the areas shielded from UV light. We visualized the patterns by immersing them in silver nitrate aqueous solution overnight (Figure 4A,B, bright field image).
(A)
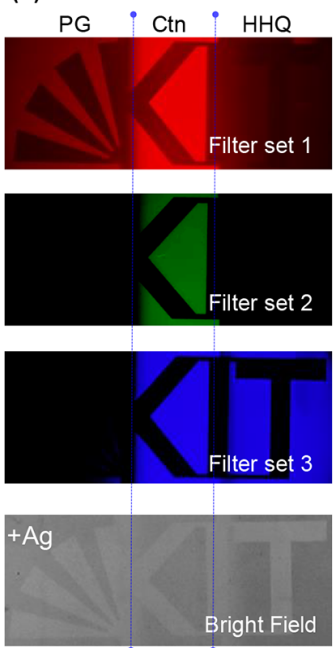

(C)

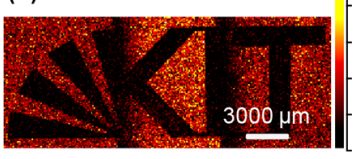

165.06 u,MC: 16, TC: $1.149 \mathrm{e}+5$

(B)

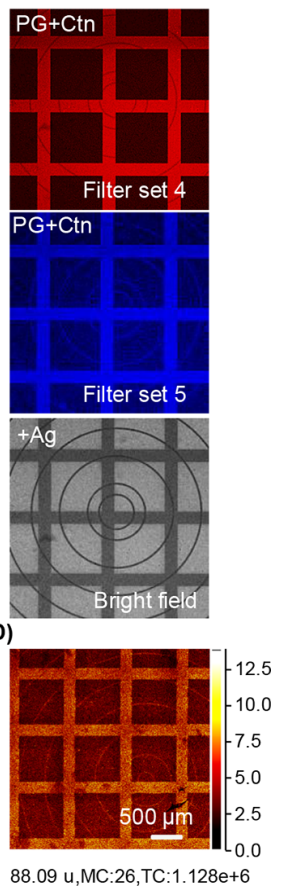

Figure 4. Sequential patterning of polyphenols in the presence of SA at $\mathrm{pH} 8.0$ after UV irradiation through a photomask for $10 \mathrm{~min}$. (A) Left part of the KIT logo was prepared using PG solution modified with Rhodamine 110; "K" and "T" letters, middle and right part of the logo, were made using $\mathrm{Ctn}$ and HHQ solutions, respectively. Filter sets 1,2 , and 3 were used to visualize the different coatings. Bright field microscopy images of the "KIT" pattern modified with silver particles (bottom). (B) Overlaid pattern formed on the surface by first UV irradiating PG solution through a photomask with circular patterns, followed by washing and Rhodamine modification. The second pattern was formed on the surface by UV irradiating the Ctn solution through a photomask with square patterns. Fluorescence microscopy images were taken using filter sets 4 and 5 . Bright field microscopy images of the silver modified pattern (bottom). (C, D) ToF SIMS analysis confirms the deposition and distribution of the phenolic mass fragments on the surface. (The KIT logo was used with permission from Karlsruhe Institute of Technology, Germany.)

An interesting application of our method is to create complex overlaid patterns of different phenolic compounds (Figure 4B). Bright field microscopy, fluorescence, and $\mathrm{X}$ ray photoelectron spectroscopy (XPS) analysis (Figure S23) clearly indicate the ability to postmodify the polyphenolic micropatterns.

Last but not least, we demonstrated the ability to create patterns of phenolic compounds (PG $0.2 \mathrm{mg} / \mathrm{mL}$, phosphate buffer $5 \mathrm{mmol} / \mathrm{L}$, $\mathrm{pH} 8.0$, SA/PG molar ratio $0.4: 1,10 \mathrm{~min}$ of UV irradiation through a quartz photomask) inside of microfluidic channels (Figure 5A) and formation of gradients of the polyphenolic coatings using a moving mask (Figure $5 B, C)$. The phenolic pattern inside the capillary and the gradient patterns were postmodified with silver particles or Rhodamine dye (Figure 5). Such flexibility in the spatiotem poral control of the deposition of different phenolic compounds will be useful in a variety of applications in biotechnology, microfluidics, and surface functionalization.

\section{CONCLUSION}

We introduced a new strategy to efficiently control the polymerization and deposition of natural plant derived phenolic 

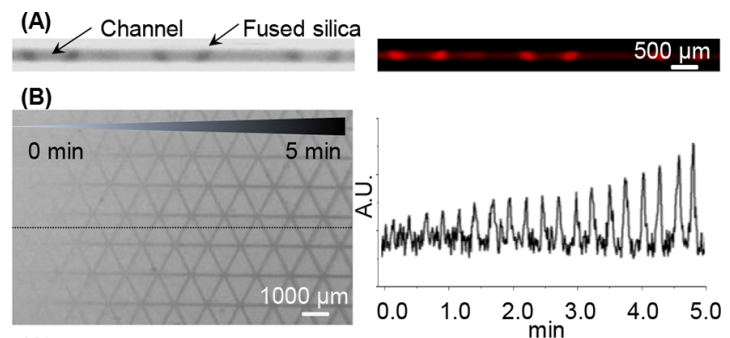

(C)
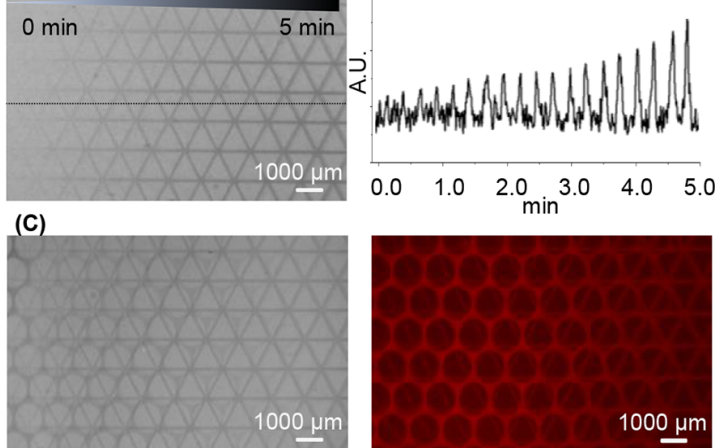

Figure 5. Polyphenolic pattern from PG solution ( $\mathrm{pH}$ 8.0) containing SA (SA/PG molar ratio of 0.4:1) was made (A) inside the capillary by UV irradiation through a photomask for $10 \mathrm{~min}$. (B) A gradient polyphenolic pattern was introduced on the flat surface and modified with silver nanoparticles. (C) Overlaid gradient polyphenolic patterns made on the surface from PG (triangle) and Ctn (hexagonal) solutions. Bright field and fluorescence microscopy images of the silver (left) and Rhodamine modified (right) patterns.

compounds under alkaline conditions, where both polymer ization and deposition are most efficient. Natural antioxidants such as sodium ascorbate (SA), glutathione (GSH), and uric acid (UA) were used to inhibit the uncontrolled oxidation of phenolic compounds, while UV light could trigger the oxidation, polymerization, and deposition of polyphenols in a spatiotemporally controlled manner. This general phenomenon was applied to nine plant derived phenols including pyrogallol, gallic acid, caffeic acid, tannic acid, pyrocatechol, catechin, morin, hydroxyhydroquinone, and epigallocatechin gallate and was demonstrated with three natural antioxidants SA, GSH, and UA. AFM analysis demonstrates that the produced nano coatings, formed under alkaline and UV antioxidant controlled conditions, are more homogeneous than those formed without antioxidants and UV light. We show the applicability of the method to create micropatterns or gradients of polyphenolic coatings, to control the polymerization temporally and stepwise, and to form patterns inside of microfluidic channels and capillaries.

\section{Notes}

The authors declare no competing financial interest.

\section{ACKNOWLEDGMENTS}

The research was supported by the ERC Starting Grant (ID: 337077 DropCellArray), by the ERC Starting Grant (ID: 715900 Redox Shields), and by the Helmholtz Association's Initiative and Networking Fund (Grant VH NG 621). H.Z. thanks China Scholarship Coucil for PhD scholarship. The K Alpha+ instrument was financially supported by the Federal Ministry of Economics and Technology on the basis of a decision by the German Bundestag.

\section{REFERENCES}

(1) Lee, J. S.; Lee, J. S.; Lee, M. S.; An, S.; Yang, K.; Lee, K.; Yang, H S.; Lee, H.; Cho, S. W. Plant Flavonoid Mediated Multifunctional Surface Modification Chemistry: Catechin Coating for Enhanced Osteogenesis of Human Stem Cells. Chem. Mater. 2017, 29, 43754384.

(2) Björnmalm, M.; Cui, J.; Bertleff Zieschang, N.; Song, D.; Faria, M.; Rahim, M. A.; Caruso, F. Nanoengineering Particles through Template Assembly. Chem. Mater. 2017, 29, 289-306.

(3) Ejima, H.; Richardson, J. J.; Caruso, F. Metal phenolic Networks as a Versatile Platform to Engineer Nanomaterials and Biointerfaces. Nano Today 2016, 12, 136-148.

(4) Wang, Y.; Park, J. P.; Hong, S. H.; Lee, H. Biologically Inspired Materials Exhibiting Repeatable Regeneration with Self Sealing Capabilities without External Stimuli or Catalysts. Adv. Mater. 2016, 28, 9961-9968.

(5) Zhang, X.; Lv, Y.; Yang, H. C.; Du, Y.; Xu, Z. K. Polyphenol Coating as an Interlayer for Thin Film Composite Membranes with Enhanced Nanofiltration Performance. ACS Appl. Mater. Interfaces 2016, 8, 32512-32519.

(6) Tang, C.; Amin, D.; Messersmith, P. B.; Anthony, J. E.; Prad'homme, R. K. Polymer Directed Self Assembly of pH Responsive Antioxidant Nanoparticles. Langmuir 2015, 31, 3612-3620.

(7) Zhan, K.; Ejima, H.; Yoshie, N. Antioxidant and Adsorption Properties of Bioinspired Phenolic Polymers: A Comparative Study of Catechol and Gallol. ACS Sustainable Chem. Eng. 2016, 4, 3857-3863.

(8) Lu, Y. C.; Luo, P. C.; Huang, C. W.; Leu, Y. L.; Wang, T. H.; Wei, K. C.; Wang, H. E.; Ma, Y. H. Augmented Cellular Uptake of Nanoparticles using Tea Catechins: Effect of Surface Modification on Nanoparticle cell Interaction. Nanoscale 2014, 6, 10297-10306.

(9) Shin, M.; Kim, K.; Shim, W.; Yang, J. W.; Lee, H. Tannic Acid as a Degradable Mucoadhesive Compound. ACS Biomater. Sci. Eng. 2016, 2, 687-696.

(10) Yao, X. P.; Zheng, X. Y.; Zhang, J. X.; Cai, K. Y. Oxidation induced Surface Deposition of Tannic acid: Towards Molecular Gates on Porous Nanocarriers for Acid responsive Drug Delivery. RSC Adv. 2016, 6, 76473-76481.

(11) Sileika, T. S.; Barrett, D. G.; Zhang, R.; Lau, K. H. A.; Messersmith, P. B. Colorless Multifunctional Coatings Inspired by Polyphenols Found in Tea, Chocolate, and Wine. Angew. Chem., Int. Ed. 2013, 52, 10766-10770.

(12) Das, C.; Chatterjee, S.; Kumaraswamy, G.; Krishnarnoorthy, K. Elastic Compressible Energy Storage Devices from Ice Templated Polymer Gels treated with Polyphenols. J. Phys. Chem. C 2017, 121, 3270-3278

(13) Rahim, M. A.; Björnmalm, M.; Bertleff Zieschang, N.; Besford, Q.; Mettu, S.; Suma, T.; Faria, M.; Caruso, F. Rust Mediated Continuous Assembly of Metal-Phenolic Networks. Adv. Mater. 2017, 29, 1606717.

(14) Rahim, M. A.; Björnmalm, M.; Bertleff Zieschang, N.; Ju, Y.; Mettu, S.; Leeming, M. G.; Caruso, F. Multiligand Metal-Phenolic Assembly from Green Tea Infusions. ACS Appl. Mater. Interfaces, in press, 2017, DOI: 10.1021/acsami.7b09237.
Nicolas Plumeré: 0000000253037865

Pavel A. Levkin: 000000025975 948X

\section{Corresponding Author}

*E mail: levkin@kit.edu.

Farid Behboodi-Sadabad: 0000000172468567 
(15) Rahim, M. A.; Kempe, K.; Mullner, M.; Ejima, H.; Ju, Y.; van Koeverden, M. P.; Suma, T.; Braunger, J. A.; Leeming, M. G.; Abrahams, B. F.; Caruso, F.; Müllner, M.; Ejima, H.; Ju, Y.; van Koeverden, M. P.; Suma, T.; Braunger, J. A.; Leeming, M. G.; Abrahams, B. F. Surface Confined Amorphous Films from Metal Coordinated Simple Phenolic Ligands. Chem. Mater. 2015, 27, 58255832 .

(16) Rahim, M. A.; Björnmalm, M.; Suma, T.; Faria, M.; Ju, Y.; Kempe, K.; Müllner, M.; Ejima, H.; Stickland, A. D.; Caruso, F. Metal-Phenolic Supramolecular Gelation. Angew. Chem., Int. Ed. 2016, 55, 13803-13807.

(17) Conte, M. P.; Lau, K. H. A.; Ulijn, R. V. Biocatalytic Self Assembly Using Reversible and Irreversible Enzyme Immobilization. ACS Appl. Mater. Interfaces 2017, 9, 3266-3271.

(18) Zhang, S. H.; Jiang, Z. Y.; Wang, X. L.; Yang, C.; Shi, J. F. Facile Method To Prepare Microcapsules Inspired by Polyphenol Chemistry for Efficient Enzyme Immobilization. ACS Appl. Mater. Interfaces 2015, 7, 19570-19578.

(19) Dhand, C.; Harini, S.; Venkatesh, M.; Dwivedi, N.; Ng, A.; Liu, S. P.; Verma, N. K.; Ramakrishna, S.; Beuerman, R. W.; Loh, X. J.; Lakshminarayanan, R. Multifunctional Polyphenols and Catechol amines Based Self Defensive Films for Health Care Applications. ACS Appl. Mater. Interfaces 2016, 8, 1220-1232.

(20) Barrett, D. G.; Sileika, T. S.; Messersmith, P. B. Molecular Diversity in Phenolic and Polyphenolic Precursors of Tannin inspired Nanocoatings. Chem. Commun. 2014, 50, 7265-7268.

(21) Hong, S.; Yeom, J.; Song, I. T.; Kang, S. M.; Lee, H.; Lee, H. Pyrogallol 2 Aminoethane: A Plant Flavonoid Inspired Molecule for Material Independent Surface Chemistry. Adv. Mater. Interfaces 2014, 1,1400113 .

(22) Zieger, M. M.; Pop Georgievski, O.; Pereira, A. D.; Verveniotis, E.; Preuss, C. M.; Zorn, M.; Reck, B.; Goldmann, A. S.; Rodriguez Emmenegger, C.; Barner Kowollik, C. Ultrathin Monomolecular Films and Robust Assemblies Based on Cyclic Catechols. Langmuir 2017, 33, 670-679.

(23) Park, M.; Shin, M.; Kim, E.; Lee, S.; Park, K. I.; Lee, H.; Jang, J. H. The Promotion of Human Neural Stem Cells Adhesion using Bioinspired Poly (norepinephrine) Nanoscale Coating. J. Nanomater. 2014, 2014, 1 .

(24) Kim, E.; Lee, S.; Hong, S.; Jin, G.; Kim, M.; Park, K. I.; Lee, H.; Jang, J. H. Sticky "Delivering From" Strategies Using Viral Vectors for Efficient Human Neural Stem Cell Infection by Bioinspired Catechol amines. ACS Appl. Mater. Interfaces 2014, 6, 8288-8294.

(25) Lim, C.; Huang, J.; Kim, S.; Lee, H.; Zeng, H.; Hwang, D. S. Nanomechanics of Poly(catecholamine) Coatings in Aqueous Solutions. Angew. Chem., Int. Ed. 2016, 55, 3342-3346.

(26) Ma, S. H.; Lee, H.; Liang, Y. M.; Zhou, F. Astringent Mouthfeel as a Consequence of Lubrication Failure. Angew. Chem., Int. Ed. 2016, 55, 5793-5797.

(27) Hong, S.; Kim, J.; Na, Y. S.; Park, J.; Kim, S.; Singha, K.; Im, G.; Han, D. K.; Kim, W. J.; Lee, H. Poly(norepinephrine): Ultrasmooth Material Independent Surface Chemistry and Nanodepot for Nitric Oxide. Angew. Chem., Int. Ed. 2013, 52, 9187-9191.

(28) Zhang, C.; Ou, Y.; Lei, W. X.; Wan, L. S.; Ji, J.; Xu, Z. K. $\mathrm{CuSO}_{4} / \mathrm{H}_{2} \mathrm{O}_{2}$ Induced Rapid Deposition of Polydopamine Coatings with High Uniformity and Enhanced Stability. Angew. Chem., Int. Ed. 2016, 55, 3054-3057.

(29) Cho, J. H.; Katsumata, R.; Zhou, S. X.; Kim, C. B.; Dulaney, A. R.; Janes, D. W.; Ellison, C. J. Ultrasmooth Polydopamine Modified Surfaces for Block Copolymer Nanopatterning on Flexible Substrates. ACS Appl. Mater. Interfaces 2016, 8, 7456-7463.

(30) Forooshani, P. K.; Lee, B. P. Recent Approaches in Designing Bioadhesive Materials Inspired by Mussel Adhesive Protein. J. Polym. Sci., Part A: Polym. Chem. 2017, 55, 9-33.

(31) Qiu, W. Z.; Zhong, Q. Z.; Du, Y.; Lv, Y.; Xu, Z. K. Enzyme triggered Coatings of Tea Catechins/Chitosan for Nanofiltration Membranes with High Performance. Green Chem. 2016, 18, 62056208.
(32) Lee, B. P.; Dalsin, J. L.; Messersmith, P. B. Synthesis and Gelation of DOPA Modified Poly(ethylene glycol) Hydrogels. Biomacromolecules 2002, 3, 1038-1047.

(33) Ejima, H.; Richardson, J. J.; Liang, K.; Best, J. P.; van Koeverden, M. P.; Such, G. K.; Cui, J. W.; Caruso, F. One Step Assembly of Coordination Complexes for Versatile Film and Particle Engineering. Science 2013, 341, 154-157.

(34) Du, X.; Li, L.; Behboodi Sadabad, F.; Welle, A.; Li, J.; Heissler, S.; Zhang, H.; Plumeré, N.; Levkin, P. A. Bio inspired Strategy for Controlled Dopamine Polymerization in Basic Solutions. Polym. Chem. 2017, 8, 2145-2151.

(35) Behboodi Sadabad, F.; Zhang, H.; Trouillet, V.; Welle, A.; Plumeré, N.; Levkin, P. A. UV Triggered Polymerization, Deposition, and Patterning of Plant Phenolic Compounds. Adv. Funct. Mater. 2017, $27,1700127$.

(36) Du, X.; Li, L. X.; Li, J. S.; Yang, C. W.; Frenkel, N.; Welle, A.; Heissler, S.; Nefedov, A.; Grunze, M.; Levkin, P. A. UV Triggered Dopamine Polymerization: Control of Polymerization, Surface Coat ing, and Photopatterning. Adv. Mater. 2014, 26, 8029-8033.

(37) Wang, L.; Shi, Y.; Chen, S.; Wang, W.; Tian, M.; Ning, N.; Zhang, L. Highly Efficient Mussel like Inspired Modification of Aramid Fibers by UV accelerated Catechol/Polyamine Deposition Followed Chemical Grafting for High performance Polymer Composites. Chem. Eng. J. 2017, 314, 583-593.

(38) Yuan, Z.; Zhao, Y.; Yang, W.; Hu, Y.; Cai, K.; Liu, P.; Ding, H. Fabrication of Antibacterial Surface via UV inducing Dopamine Polymerization Combined with Co deposition $\mathrm{Ag}$ Nanoparticles. Mater. Lett. 2016, 183, 85-89.

(39) Bai, G.; Ma, S.; Qie, R.; Liu, Z.; Shi, Y.; Li, C.; Wang, R.; Guo, X.; Zhou, F.; Jia, X. UV Triggered Surface Initiated Polymerization from Colorless Green Tea Polyphenol Coated Surfaces. Macromol. Rapid Commun. 2016, 37, 1256-1261.

(40) Cencer, M.; Liu, Y.; Winter, A.; Murley, M.; Meng, H.; Lee, B. P. Effect of $\mathrm{pH}$ on the Rate of Curing and Bioadhesive Properties of Dopamine Functionalized Poly(ethylene glycol) Hydrogels. Biomacro molecules 2014, 15, 2861-2869.

(41) Quideau, S.; Deffieux, D.; Douat Casassus, C.; Pouysegu, L. Plant Polyphenols: Chemical Properties, Biological Activities, and Synthesis. Angew. Chem., Int. Ed. 2011, 50, 586-621.

(42) Bendary, E.; Francis, R. R.; Ali, H. M. G.; Sarwat, M. I.; El Hady, S. Antioxidant and Structure-activity Relationships (SARs) of some Phenolic and Anilines Compounds. Ann. Ann. Agric. Sci. 2013, 58, 173-181.

(43) Evmiridis, N. P.; Vlessidis, A. G.; Thanasoulias, N. C. Chemical Analysis Through CL detection Assisted by Periodate Oxidation. Bioinorg. Bioinorg. Chem. Appl. 2007, 2007, 1.

(44) Danilewicz, J. C. Interaction of Sulfur Dioxide, Polyphenols, and Oxygen in a Wine model System: Central Role of Rron and Copper. Am. J. Enol. Vitic. 2007, 58 (1), 53-60.

(45) Waterhouse, A. L.; Laurie, V. F. Oxidation of Wine Phenolics: A Critical Evaluation and Hypotheses. Am. J. Enol. Vitic. 2006, 57 (3), 306-313.

(46) Lu, J. M.; Lin, P. H.; Yao, Q. Z.; Chen, C. Y. Chemical and Molecular Mechanisms of Antioxidants: Experimental Approaches and Model Systems. J. Cell. Mol. Med. 2010, 14, 840-860.

(47) Szultka, M.; Buszewska Forajta, M.; Kaliszan, R.; Buszewski, B. Determination of Ascorbic Acid and its Degradation Products by High performance Liquid Chromatography triple Quadrupole Mass Spectrometry. Electrophoresis 2014, 35, 585-592.

(48) Stinefelt, B.; Leonard, S. S.; Blemings, K. P.; Shi, X. L.; Klandorf, H. Free Radical Scavenging, DNA Protection, and Inhibition of Lipid Peroxidation Mediated by Uric Acid. Ann. Clin. Lab. Sci. 2005, 35 (1), $37-45$.

(49) Galano, A.; Raul Alvarez Idaboy, J. Glutathione: Mechanism and Kinetics of its Non enzymatic Defense Action Against Free Radicals. RSC Adv. 2011, 1, 1763-1771.

(50) Nimse, S. B.; Pal, D. Free Radicals, Natural Antioxidants, and Their Reaction Mechanisms. RSC Adv. 2015, 5, 27986-28006. 
(51) Waring, W. S. Uric Acid: An Important Antioxidant in Acute Ischaemic Stroke. QJM 2002, 95, 691-693.

(52) Thiagarajan, S.; Tsai, T. H.; Chen, S. M. Easy Modification of Glassy Carbon Electrode for Simultaneous Determination of Ascorbic Acid, Dopamine and Uric acid. Biosens. Bioelectron. 2009, 24, 27122715.

(53) Geyer, F. L.; Ueda, E.; Liebel, U.; Grau, N.; Levkin, P. A. Superhydrophobic Superhydrophilic Micropatterning: Towards Ge nome on a Chip Cell Microarrays. Angew. Chem., Int. Ed. 2011, 50, 8424-8427.

(54) Parry, K. L.; Shard, A. G.; Short, R. D.; White, R. G.; Whittle, J. D.; Wright, A. ARXPS Characterisation of Plasma Polymerised Surface Chemical Gradients. Surf. Interface Anal. 2006, 38, 1497-1504.

(55) Scofield, J. H. Hartree Slater Subshell Photoionization Cross Sections at 1254 and 1487EV. J. Electron Spectrosc. Relat. Phenom. 1976, 8, 129-137.

(56) Talamond, P.; Verdeil, J. L.; Conejero, G. Secondary Metabolite Localization by Autofluorescence in Living Plant Cells. Molecules 2015, 20, 5024-37.

(57) Roshchina, V. V. Vital Autofluorescence: Application to the Study of Plant Living Cells. Int. J. Spectrosc. 2012, 2012, 1. 
Karlsruher Institut für Technologie

\section{Repository KITopen}

Dies ist ein Postprint/begutachtetes Manuskript.

Empfohlene Zitierung:

Behboodi-Sadabad, F.; Zhang, H.; Trouillet, V.; Welle, A.; Plumeré, N.; Levkin, P. A.

Bioinspired Strategy for Controlled Polymerization and Photopatterning of Plant Polyphenols. 2018. Chemistry of materials, 30.

DOI: $10.5445 / / R / 1000082428$

Zitierung der Originalveröffentlichung:

Behboodi-Sadabad, F.; Zhang, H.; Trouillet, V.; Welle, A.; Plumeré, N.; Levkin, P. A.

Bioinspired Strategy for Controlled Polymerization and Photopatterning of Plant Polyphenols. 2018. Chemistry of materials, 30 (6), 1937-1946.

DOI:10.1021/acs.chemmater.7b04914 